\title{
Belphégor
}

\section{Le genre comme pratique historique}

\section{Matthieu Letourneux}

\section{OpenEdition}

\section{Journals}

Electronic version

URL: http://journals.openedition.org/belphegor/732

DOI: 10.4000/belphegor.732

ISSN: 1499-7185

\section{Publisher}

LPCM

\section{Electronic reference}

Matthieu Letourneux, « Le genre comme pratique historique », Belphégor [Online], 14 | 2016, Online since 24 October 2016, connection on 20 April 2019. URL : http://journals.openedition.org/ belphegor/732 ; DOI : 10.4000/belphegor.732

This text was automatically generated on 20 April 2019.

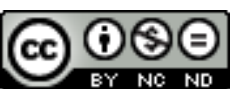

Belphégor est mis à disposition selon les termes de la Licence Creative Commons Attribution - Pas d'Utilisation Commerciale - Pas de Modification 4.0 International. 


\title{
Le genre comme pratique historique
}

\author{
Matthieu Letourneux
}

\section{Problèmes de l'approche formaliste des genres médiatiques}

1 Les études de Lettres Modernes qui portent sur les genres tendent à privilégier une interrogation sur les genres littéraires hérités d'Aristote (épopée, drame, dithyrambe) plutôt que celle, pourtant plus en accord avec l'usage courant du terme, sur les genres médiatiques ou populaires (roman policier, d'aventures, sentimental). Ce choix a eu une incidence majeure sur la façon qu'ont eu les chercheurs de penser les productions médiatiques. En effet, plaquant le modèle formaliste et abstrait qui a prévalu pour les genres littéraires, ils ont eu tendance à approcher les genres médiatiques dans une même perspective formaliste, cherchant à définir le roman policier, la science-fiction ou le roman d'aventures comme des catégories transcendantes susceptibles de décrire un ensemble de textes possédant tous les mêmes traits. Souvent, cette posture était assortie de précautions oratoires visant à écarter les définitions éditoriales ou commerciales comme imprécises ou inauthentiques. Ainsi oppose-t-on la scientificité de la démarche de l'Universitaire et sa faculté à établir des catégories transcendantes aux pratiques courantes des acteurs de l'espace culturel et médiatique: la presse, la critique médiatique, les éditeurs, les auteurs populaires et, bien sûr le grand public, tous se voient reprocher de ne pas s'appuyer sur des critères définitoires objectifs, cohérents et universels, et d'être commandés par des intérêts propres brouillant l'objectivité de leur propos.

2 Passons sur la conception de la littérature et de la culture qu'une telle posture suppose. Soulignons en revanche la contradiction logique sur laquelle repose la démarche. En effet, si, pour décrire des genres médiatiques de façon objective on doit s'arracher à l'interdiscours de la société qui emploie ces notions, à quelle définition parvient-on en optant pour cette démarche? Que reste-t-il de la dimension médiatique de ces genres dès l'instant où on les a soustraits à leur contexte médiatique ? Pour le dire autrement, un 
genre médiatique existe-t-il indépendamment du discours social et de ses formulations médiatiques? Plus largement, existe-t-il des catégories culturelles indépendantes des cultures qui les produisent? Il nous semble évident que non. Dès lors, si l'on peut s'écarter du contexte discursif et social pour forger des catégories génériques/analytiques (comme lorsqu'on évoque le roman de remariage ou la dystopie), il est beaucoup plus problématique de le faire à propos de genres dont l'origine est culturelle et médiatique : pourquoi prétendre que le roman policier, la science-fiction, la chick lit ou l'anticipation gagnent à être pensés à travers une définition transcendante s'ils sont de fait le produit d'un interdiscours situé dans le temps et l'espace ? Pourquoi repousser les usages, si dans le fond ce sont ces usages qui ont imposé, au fil du temps et de la variation sérialisée des textes, un concept qui auparavant n'existait pas indépendamment d'eux? Plus fondamentalement, si l'on s'accorde pour considérer que la culture est le résultat des pratiques, des représentations et des discours d'une société, et de leur sédimentation au fil du temps en un héritage culturel, si en outre on considère qu'un genre médiatique est le produit d'une société et donc le résultat d'une culture, alors il n'y a pas d'autre définition valide que celles formulées par une société au fil du temps et des usages. C'est d'autant plus vrai que les productions littéraires ne sont que du discours et n'existent pas hors de celui-ci.

On peut le montrer à partir de l'exemple des termes employés pour définir ce que l'on rassemble autour de la notion anachronique d'anticipation (comme anticipation de l'avenir) ${ }^{1}$. On trouve certes des expressions comme « roman scientifique » (fréquent dans les années 1880) et "roman d'anticipation » (dès 1910 et massivement à partir des années $1920^{2}$ ), mais on rencontre également, pour des œuvres qu'on définirait aujourd'hui comme de l'anticipation, des termes comme "roman philosophique» (employé par exemple à propos de La Race future de Bullwer-Lytton), «roman d'aventures fantastiques» (utilisé par la critique aussi bien à propos de Jules Verne que de Wells), " merveilleux logique ", etc. On croise encore des termes renvoyant à d'autres modèles de généricité: «fantaisie» (Clément Vautel, Les Évadés du progrès, 1919), «conte physiologique " (Henry Beaunis, Madame Mazurel, 1895), "conte» (Richepin, 1922), "Grand roman mystérieux » (Perrin et H. Lanos, 1910-1911), « conte futur » (Richepin, 1897) et cent autres formulations. On voit combien cette hétérogénéité terminologique met en cause l'unité a posteriori de l'anticipation. Car les récits se prêtent fort bien à une telle distribution dans d'autres ensembles génériques, différente de celle, anachronique, que nous avons tendance à privilégier. La multiplication des sous-titres génériques employés témoigne soit que l'œuvre est associée à d'autres séries génériques (celles du merveilleux ou du roman à idées en particulier), soit qu'elle n'invite pas à une lecture orientée par une série architextuelle qui en médiatiserait la réception (cas des sous-titres fantaisistes visant à insister sur un effet d'hapax). Présenter le roman de Bullwer-Lytton comme un roman philosophique, c'est insister sur sa relation avec d'autres récits du même genre, avec comme modèles lointains Rousseau ou Voltaire.

On peut prendre pour exemple le cas du "conte futur », expression employée par Jean Richepin pour désigner le Dernier inventeur dans son édition de la nouvelle en livre en 1897. La préoriginale est publiée dans le Gil Blas en 1889 sans que le terme n'apparaisse encore. Le Gil Blas offrait souvent de ces petites nouvelles excentriques et paradoxales qualifiées de contes et écrites par des auteurs à la mode, comme Maupassant ou Alphonse Allais. C'est exactement à cette catégorie qu'appartient le texte de Richepin. Le récit oppose un monde futuriste entièrement technologique au "dernier inventeur », homme 
qui, ô scandale, a inventé le lyrisme, le pain et le vin. On voit comment ce récit s'inscrit dans une posture artistique négociant avec la logique médiatique (l'artiste joue le jeu du support mais affirme dans l'œuvre son irréductible marginalité). L'intitulé « conte futur » indique bien le genre (celui du " conte", terme employé pour désigner en particulier ce modèle de nouvelles à pointe, fondées sur l'habileté du conteur ${ }^{3}$, le terme « futur » visant à apporter une précision thématique à valeur d'accroche). L'œuvre s'inscrit explicitement dans la série architextuelle des nouvelles publiées dans la presse. On voit comment, en l'appréhendant immédiatement comme un récit d'anticipation, on donne une valeur anachronique à un texte dont on ne conserve que le vernis thématique, loin de la signification collective qui le portait, celui du récit bref, virtuose, lié au rythme de lecture de la presse, mettant en scène la voix de l'auteur (le conteur) et son habileté, au point ici de définir une posture artiste.

\section{Problèmes de l'Histoire axiologique}

L'approche formaliste perd ainsi de vue la fonction communicationnelle des genres, laquelle ne peut s'appréhender que dans l'interdiscours. Mais quand on écarte l'approche abstraite des genres, on rencontre alors un second écueil, plus fréquent encore, au point de se retrouver dans la plupart des études sur les genres populaires et médiatiques aujourd'hui. Contre la perspective transcendante, bien des chercheurs vont en effet faire le choix d'une Histoire du genre - du roman policier, de la science-fiction, de la littérature fantastique, etc. Généralement, les travaux sur le sujet distinguent une période archaïque, celle des prémisses du genre, en se référant à des auteurs clés, présentés comme des précurseurs : Conan Doyle pour le roman policier, Jules Verne et H. G. Wells pour la science fiction, etc. Et pour chaque génération, l'importance des auteurs est évaluée à partir de leur fortune, autrement dit, suivant une perspective téléologique. Bref, ces livres font une Histoire littéraire du genre - ou, devrait-on dire, reprenant l'expression de Paul Veyne dans Comment on écrit l'Histoire, une Histoire littéraire axiologique du genre ${ }^{4}$. A travers la notion d'axiologie, Paul Veyne veut souligner que l'Histoire littéraire est dominée par la question de la valeur des œuvres. Or, dans la mesure où celle-ci suppose une évaluation, et donc la mise en avant d'une réception, alors l'Histoire littéraire est axiologique parce qu'elle appréhende les œuvres à partir de hiérarchies définies $a$ posteriori, depuis le présent de la réception. Ouvrir une Histoire de la littérature, ce n'est pas ouvrir un livre d'Histoire, puisque ce qui est décrit n'est pas la situation des productions littéraires dans une période donnée, mais un ensemble d'œuvres sélectionnées à partir d'un héritage contemporain, les œuvres importantes pour nous. Dès lors, l'Histoire littéraire axiologique est, fondamentalement, une Histoire anachronique.

6 Cette approche anachronique caractérise encore très souvent les études consacrées aux genres populaires et médiatiques. Or, elle pose un problème pour l'étude de l'œuvre. Parler d'« ancêtres du genre", de précurseurs ou d'auteurs fondateurs, à propos, par exemple de Jules Verne par rapport à la science-fiction, c'est offrir une lecture anachronique de cet auteur, et évoquer des pratiques culturelles antérieures à leur existence même. De même, définir des grands auteurs du genre, c'est le plus souvent mettre l'accent sur leur fortune, donc sur leur descendance, définissant a posteriori leur place et leur signification. Or, ce qui est déjà problématique dans la littérature en général, l'est davantage encore dans les productions sérielles (et donc dans la littérature de genre). Par définition, un auteur sériel entre en dialogue avec des séries de textes antérieures et avec 
les architextes qu'elles constituent. Quand Edgar Rice Burroughs écrit A Princess of Mars en 1917, il ne cherche pas à fonder le space opera qui n'est évidemment rien pour lui. Il cherche simplement à capitaliser sur le succès d'une série constituée antérieurement, le lost race tale, dont le référent prototypique est à l'époque Henry Rider Haggard. L'amour d'un Occidental pour une princesse d'une civilisation inconnue est en effet au cœur des aventures d'Allan Quatermain ou du cycle de She. Ce n'est pas tant Burroughs qui fonde le space opera, mais ses épigones et relecteurs, qui en font un référent prototypique de leur discours 5 .

7 Prenons un exemple caractéristique de la torsion que peut produire une telle lecture anachronique dans l'appréhension des œuvres. Penser Jules Verne dans sa relation à l'anticipation, c'est isoler un faible nombre de ses œuvres de l'ensemble des «Voyages extraordinaires » et briser ainsi l'unité discursive de son propos, qui tire sa signification de sa situation éditoriale et communicationnelle. Du même coup cela contraint à faire de même pour certains de ses épigones : par exemple Paul d'Ivoi (pas d'éléments imaginaires dans Les Cinq sous de Lavarède) et Louis Boussenard (rares sont ses romans qui peuvent être qualifiés d'anticipation). Or, les uns comme les autres se pensent dans des séries architextuelles qui sont celles de la littérature pour la jeunesse (avec la Comtesse de Ségur) et de la vulgarisation scientifique (avec Flammarion ou Macé et la «bouchée de pain »). Par ailleurs, le genre dans lequel ils inscrivent leurs œuvres est celui du roman d'aventures géographiques ( «roman de voyage » - c'est ce que disent les sous-titres et surtitres). Cela explique le fait que, dans ces œuvres, le chronotope privilégié est celui du présent de l'œuvre voire, selon les conventions romanesques, celui d'un passé très récent. Écrit en 1864, Voyage au centre de la terre s'ouvre en 1863 ; Vingt mille lieues sous les mers débute en 1866, soit quatre ans avant la date de rédaction du roman, et De la terre à la lune se déroule peu après la fin de la Guerre de Sécession; et si la plupart des romans de Verne ne semblent pas indiquer de date précise, ils font référence à une actualité qui les pose très clairement comme contemporains. De même, les dates que cite Paul d'Ivoi renvoient presque toutes à un passé récent, faisant de ses voyages excentriques des fictions d'actualité. Enfin Les Secrets de Monsieur Synthèse de Boussenard (1888) débute en 1884. Autrement dit, la logique des auteurs est à l'opposé de celle d'un récit d'anticipation. Les y réduire revient à manquer la nature de leurs propos.

Plus gênant encore, l'approche anachronique tend à substituer l'homogénéité des discours sociaux du temps de la réception (le nôtre) aux imaginaires de l'époque. Dans notre cas par exemple, on évoque souvent une unité de la première anticipation autour de l'idée d'un merveilleux scientifique. Mais on évacue un des domaines importants de ce merveilleux scientifique qu'est l'imaginaire spirite. Celui-ci nourrit pourtant tout à la fois des œuvres comme celle de Théophile Gautier (Spirite) ou de Villiers de l'Isle Adam (L'Eve future) à l'intersection des techniques scientifiques et du spiritisme, ou encore Le Château des Carpathes de Jules Verne (dont la signification repose justement sur l'opposition entre deux types d'émerveillement scientifique). Et la question de son exclusion ou non du champ de la recherche est un enjeu majeur. On rappellera d'ailleurs que l'expression « roman scientifique » a servi à désigner un type de pseudo-science avant de désigner un genre de romans (les romans verniens). Quand, en 1845, on écrit qu'il est possible que la doctrine de Gall devienne une science, mais qu'aujourd'hui, ce n'est qu'un « roman scientifique ", on fait de l'expression un désignatif de discours savants non démontrés, pas des fictions romanesques ${ }^{6}$. 
Bien sûr, rien n'empêche d'adopter une posture anachronique. C'est ce que souligne JeanMarie-Schaeffer lorsqu'il remarque que la généricité peut être d'ordre généalogique ou analogique ${ }^{7}$. Quand la généricité généalogique insiste sur les relations que tissent les auteurs avec d'autres œuvres, la généricité analogique rapproche des œuvres qui n'étaient à l'origine pas liées entre elles par une unité générique. Mais cette seconde perspective n'est pas moins située que la première ; simplement, elle substitue le contexte de réception, comme résultat de la sédimentation de définitions et d'usages successifs du genre, à celui des producteurs et de leurs contemporains. Autrement dit, il est tout à fait possible de se détacher du contexte direct de production de l'œuvre (ou de production du genre, c'est-à-dire des discours sur le genre à un moment donné) pour lui substituer une réception située de l'œuvre qui la rapporterait au genre, en fonction du contexte interdiscursif de la réception, mais à condition d'avoir conscience qu'on ne fait rien d'autre que de déplacer le questionnement contextuel.

Dès lors, on peut considérer chaque définition anachronique ou abstraite du genre comme une proposition située (à un moment donné et dans un lieu déterminé de l'espace interdiscursif), liée en réalité à ce contexte sociodiscursif, éminemment idéologique. Si l'on peut étudier un discours collectif et historicisé sur le genre à un moment donné, il est également possible de produire une Histoire du genre comme série de moments discursifs articulant un état synchronique (l'interdiscours et ses tensions à un moment donné) à une réalité diachronique (l'héritage des discours sur le genre) qu'il ressaisirait dans ses plis. On peut en prendre un exemple frappant, celui du steampunk comme mise en fiction, volontairement anachronique, des héritages des littératures d'imagination contemporaines - puisqu'il s'agit de ce genre, né dans les années 1980, qui offre la vision d'un XIXe siècle uchronique intégrant des éléments de science fiction ${ }^{8}$. Dans ces œuvres, le référent n'est pas tant la réalité historique que la lignée intertextuelle de la sciencefiction appréhendée du point de vue de la réception. Pour un auteur de science fiction de la fin du XXe siècle, l'origine du genre est en effet à rechercher chez des auteurs comme Jules Verne, Rider Haggard, Stevenson ou Conan Doyle et, plus largement, dans ce qu'on a pu appeler depuis le new romance ${ }^{9}$. Mais en manifestant cette lignée dans la diégèse, les auteurs convertissent fictionnellement en science-fiction des œuvres qui n'en étaient pas, exhibant ainsi dans le récit le travail de reconstruction du sens produit progressivement par la réception sédimentée des textes. Dans ce cas, la réception historicise son propre présent, mettant en évidence le fait que la généricité lectoriale n'est rien d'autre que la reformulation synchronique d'un héritage sédimenté.

11 On comprend que, dans la perspective sérielle, généricité lectoriale et généricité auctoriale ne sont pas des réalités hétérogènes : il n'y a d'auteur se situant dans un genre que dans la mesure où il se fait relecteur d'un héritage. Cet héritage n'est pas une réalité transcendante, mais une construction située, celle d'une réception définie par la position du locuteur (auteur, lecteur, critique, éditeur, journaliste, publicitaire, etc.), par sa façon de dialoguer avec les discours sur le genre (et en particulier ceux avec lesquels il est appelé à interagir directement - les séries génériques, médiatiques, discursives, culturelles etc. qu'il investit), par sa relation aux héritages, et par son expérience du genre (des œuvres et des définitions). Quant aux définitions dominantes du genre à une époque donnée, elles sont le résultat de cette multitude synchronique et diachronique de définitions qui coexistent dans un dialogue et un rapport de forces constants, un résultat contradictoire, trouble et problématique dont on gagne à conserver les contradictions, pour en saisir la signification. 


\section{Lieux de définition du genre} entre différents acteurs. Chaque œuvre correspond à une instanciation (puisqu'elle est seconde) de cette réalité interdiscursive, et en offre une définition implicite (puisque sa situation dans un réseau de séries intertextuelles et architextuelles tend à produire une telle définition implicite).

Bien sûr, la relation au genre transparaît avant tout dans les titres, sous-titres, les références à des auteurs ou des œuvres à valeur prototypique (dans notre cas, suivant les cas, Verne, Wells, Maurice Renard, etc.) et, de façon plus diffuse, dans le recours à des procédés stylistiques, à des types de personnages ou à des scénarios intertextuels à valeur codante. Mais à côté de cette mise en réseau produite dans les textes par les auteurs euxmêmes, la définition du genre est aussi le fait de discours allogènes: éditeur, publicitaires, critiques, journalistes peuvent ainsi associer une œuvre à un genre ou identifier un phénomène architextuel. Mais par-delà ces acteurs majeurs officiels intervenant dans la définition du genre à travers des productions textuelles identifiables, chaque lecteur, chaque consommateur, participe à son niveau à la définition du genre. De fait, si le public «commun » ne matérialise pas cette évaluation dans des productions textuelles ou médiatiques, il passe son temps, dans la parole quotidienne, à rapporter des textes à des séries architextuelles, contribuant dans ses conversations courantes à imposer des définitions intuitives des genres. Dès lors, moins influent de façon isolée que les productions des acteurs médiatiques et culturels, le discours collectif quotidien joue un rôle majeur dans l'identification et la nomination des genres et de leur institution. C'est d'ailleurs parce que les genres sont une réalité interdiscursive globale, par-delà les seules productions médiatiques et culturelles, qu'ils apparaissent comme des lieux où s'articulent les tensions du discours social - nous y reviendrons.

Il convient de remarquer que cette masse de discours allogènes - l'ensemble des discours directs ou indirects portant explicitement ou implicitement sur le genre - ne forme pas un tout homogène. Leur influence dépend du poids respectif des acteurs - de leur influence dans le champ culturel et médiatique. Quant à leur nature, elle dépend de la position et des intérêts de ceux qui les formulent, de la série discursive dans laquelle ils s'inscrivent (article critique, publicité, causerie, affiche, conversation privée), de la fonction du discours, etc. Ainsi, un texte critique sera dominé par une logique de rapprochement à des fins descriptives et explicatives. Soit il s'agira de rapprocher des œuvres similaires pour dégager une tendance, soit, parlant d'un texte que le public n'aura pas lu, le critique assortira le trait générique à des détails signifiant: on évoquera un roman à la H. G. Wells ou à la Jules Renard (suivant une logique de prototypicité); on décrira une œuvre comme un roman d'anticipation ou un roman philosophique, en développant un propos visant à justifier la relation architextuelle, dans une dynamique descriptive. De son côté, le texte publicitaire utilisera les références génériques suivant une rhétorique de séduction : « une œuvre digne de Jules Verne », « un grand roman d'anticipation » (en cherchant à convoquer un architexte le plus large possible afin de séduire le plus grand nombre); une présentation de collection par un éditeur multipliera les références prototypiques (grands auteurs à valeur de parangons) pour établir une unité signifiante et un horizon d'attente, permettant de décrire l'ensemble des œuvres. On voit que l'usage

Belphégor, 14 | 0000 
du genre se traduira par une conception plus ou moins extensive, par un mouvement de spécification ou de généralisation, etc.

Dès lors, ce que nous appelons le genre est le résultat d'un réseau complexe de discours en interaction et en tension dont les échanges et les contradictions produisent un effet d'unité problématique. De fait, il existe des cohérences discursives secondes, liées par exemple au média, à des logiques communicationnelles, à des positions idéologiques, à la proximité des thèmes et des structures narratives avec d'autres séries architextuelles... autant de situation qui produisent des unités sérielles qui ne sont pas nécessairement identifiées comme des genres, mais qui orientent les attentes des consommateurs.

La structuration des genres en séries architextuelles secondes permet l'existence d'ensembles cohérents d'œuvres qui ne sont pas clairement identifiés à travers un genre institué. Pensons par exemple aux récits de guerre future qui se développent massivement après 1870, et dont la signification ne peut se saisir que si on les associe aux séries textuelles formées par les différents types de productions associées au discours social patriotique et revanchard ${ }^{10}$. De même existe-t-il des unités sérielles associées à des logiques médiatiques qui définissent des traits architextuels forts sans nécessairement être identifiées à des genres : c'est le cas par exemple des cartonnages d'étrennes, des fascicules pour la jeunesse, des collections populaires. A chaque fois, la nature du public, les spécificités de la communication et les modalités de consommation déterminent des cohérences architextuelles propres, et donc des attentes spécifiques, sans pour autant renvoyer à un genre déterminant, mais qui s'articulent souvent autour de relais intertextuels à valeur prototypique (par exemple Jules Verne pour le cartonnage, Louis Boussenard pour les collections populaires pour la jeunesse, Frank Reade aux États-Unis pour les dime novels d'inventeurs de génie, H. G. Wells pour les publications dans des collections middlebrow, etc.).

17 Mais si de telles conventions architextuelles liées aux conditions matérielles de production et de réception des œuvres définissent par avance une posture auctoriale et un horizon d'attente, elles ne sont pas clairement structurées en genres. On pourrait dire ainsi qu'il existe une gradation dans la communication sérielle conduisant de séries de stéréotypes peu structurés au genre institué en passant toute un ensemble d'effets d'architextualité plus ou moins clairement établis. Dans le cas où les logiques architextuelles sont plus diffuses, il importe cependant de trancher entre ce qui est réellement un phénomène sériel (donc le résultat d'une formalisation d'un architexte dans l'interdiscours) et ce qui apparaît a posteriori sous l'effet de l'évolution de la réception, comme une réalité architextuelle, laquelle n'était perçue à l'époque. Un texte dystopique s'inscrit-il toujours dans une sérialité de la dystopie ? Ce n'est pas certain. Il est plus probable que certaines œuvres aient été perçues comme des textes philosophiques, d'autres comme des fantaisies romanesques, d'autres encore comme des romans d'aventures extraordinaires.

\section{Le genre dans l'interdiscours - remarques méthodologiques}

18 Ainsi le genre est-il le résultat de l'interdiscours, manifesté en particulier par la masse des productions médiatiques, du livre au journal, de l'affiche au jouet, du théâtre au cinéma, mais aussi par l'ensemble des conversations quotidiennes. En outre, chacune des 
définitions qui sont produites par ces différents textes et discours est liée au contexte et à la fonction pragmatique qui sont les leurs. La première conséquence méthodologique qu'il faut en tirer, c'est qu'il faut modifier radicalement le périmètre du corpus des études littéraires. En effet, si l'appréhension du genre est le résultat d'une mosaïque de définitions sérielles situées (suivant les supports de diffusion, leur mode de consommation, les cohérences médiatiques et idéologiques), alors il convient de saisir l'œuvre non dans sa seule textualité, mais dans la matérialité de sa communication. Le texte existe par rapport à un support (et aux autres textes qui partagent ce même support) ${ }^{11}$. Ledit support doit se penser en termes de contraintes de production et de modes de consommation, non seulement dans une perspective communicationnelle, mais aussi dans le réseau de sociabilités qui le porte (ce qu'avait montré Howard Becker ${ }^{12}$ ). Ainsi on ne peut comprendre les différences qui existent entre les récits de machines publiés dans les collections populaires pour la jeunesse et ceux paraissant dans les collections de cartonnages de prix et d'étrennes si on ne les ressaisit pas dans les séries médiatiques, comme ensembles de textes et comme modes de consommation qu'ils induisent: leur appréhension est liée aux mutations du système médiatique et de ses supports, lui-même déterminé par l'avènement de nouvelles pratiques de loisir et de consommation et, pour la littérature de jeunesse, par les changements intervenus dans l'éducation, autant de traits qui induisent de nouveaux usages des textes. Lectures liées à des dispositifs de transmission dans le cas des publications en cartonnages, les récits font de la machine un véhicule de savoir et d'éducation morale quand, dans les collections populaires, elle n'est plus qu'un moyen de rêverie fantastique pour l'enfant lisant seul pour le plaisir. Ces changements de contexte affectent ainsi la nature des textes et leur réception, leur relation au savoir, à la morale, les équilibres entre discursif et narratif, ou encore la structure des récits.

Or, et c'est la deuxième conséquence, il découle de cette appréhension globale un nécessaire abandon des hiérarchies qui fondent le discours littéraire. On doit absolument s'interdire de privilégier les auteurs canoniques, les grandes œuvres, les auteurs qui restent . Il s'agit au contraire de manipuler l'ensemble du corpus de textes participant du genre sans se préoccuper de la légitimité des œuvres. Alors seulement on peut substituer un autre système hiérarchique entre les œuvres, celui produit par l'interdiscours dans sa réalité synchronique et diachronique. Lorsqu'un texte évoque un genre, ou lorsqu'une œuvre est appréhendée dans sa relation à un genre, ils tendent à reformuler la relation architextuelle en termes intertextuels, autrement dit à se réclamer d'œuvres ou d'auteurs qui leur servent de modèles. Plus généralement, on saisit le genre à travers quelques figures prototypiques à partir desquelles on lui donne une identité concrète. Ce principe de prototypicité dépend de la série qui l'engage, c'est-à-dire de la position de celui qui parle : Jules Verne est davantage un référent prototypique pour les livres de jeunesse, quand Wells et Maurice Renard le sont pour la littérature plus légitimée. Certains auteurs ne sont prototypiques que durant une période brève de temps ou dans un champ sériel limité, à l'instar par exemple de Paul d'Ivoi ou de Louis Boussenard, qu'on évoque peu hors de la littérature pour la jeunesse, mais qui restent néanmoins très prototypiques dans la littérature populaire pour la jeunesse jusqu'aux années 1950. Chaque prototype hiérarchise l'appréhension du genre et distribue la cartographie des œuvres, centrales ou périphériques, qu'on lui associe. Autrement dit, il induit des traits définitoires situés qui varient suivant la position de celui qui appréhende le genre ou l'œuvre. 
20 Le rôle joué par les figures prototypiques (œuvres servant à désigner un genre) met en évidence un déplacement de l'analyse des intertextes vers les architextes. Dominée par sa préoccupation de l'œuvre et de l'auteur, l'analyse littéraire traditionnelle surinvestit la question des intertextualités sans voir que les références intertextuelles ont bien souvent pour fonction de désigner des relations architextuelles, dont les dynamiques (scénarios intertextuels, réseaux stéréotypiques) déterminent infiniment plus la signification du texte. Titrer un roman en évoquant Le Tour du monde ${ }^{13}$, c'est certes convoquer l'intertexte de Verne, mais c'est plus fondamentalement renvoyer à la série constituée des récits de voyage extraordinaire dont Verne et ses œuvres ne sont que les principaux relais prototypiques, et dont Paul d'Ivoi et Louis Boussenard seraient les prototypes secondaires. En faisant reposer la relation sérielle sur toute une série de scénarios intertextuels et de stéréotypes, les œuvres mettent en évidence combien elles obéissent fondamentalement à des logiques architextuelles. Un auteur qui choisit d'écrire dans un genre s'inspire certes souvent de modèles d'œuvres ou d'auteurs, mais il écrit surtout en fonction de conventions narratives ou thématiques qu'il associe au genre, sans les identifier à des textes précis.

21 Le quatrième déplacement analytique découle des précédents: dans la mesure où la définition du genre et ses relais prototypiques sont le résultat d'une construction interdiscursive, et dans la mesure où cette construction détermine les choix auctoriaux et la façon qu'ont les consommateurs de déchiffrer les textes, alors on est obligé de convenir que la signification de l'œuvre ne réside pas seulement dans le texte, mais autant dans le paratexte, entendu dans son sens le plus large. On peut même aller plus loin, et souligner que, lorsqu'on étudie les dynamiques sérielles, on a parfois tort de dissocier texte et paratexte. Ainsi, dans un récit publié en feuilleton dans la presse, les annonces publicitaires, le découpage en livraisons (qui n'est pas toujours, loin s'en faut, le fait de l'auteur), les références des autres articles au récit, participent tous à la signification contextuelle de l'œuvre, comme autant de façons d'orienter la lecture dans le genre. Enfin, en poussant la logique de l'interdiscours, on est obligé de convenir que la signification de l'œuvre de genre ne se situe pas tant dans le texte lui-même que dans la masse des productions auxquelles il se réfère, en amont, et de celles qui y renvoient, en aval. Nous l'avions montré à partir des «Grandes aventures d'un boy scout » de Jean de La Hire (1926), qui reprenaient à l'identique le texte de fascicules de Buffalo Bill d'avant la Première Guerre mondiale. L'auteur ne changeait pas un mot du texte, mais par la vertu du pacte de lecture générique, l'univers western devenait, pour le lecteur, un monde d'aventures spatiales ${ }^{14}$. Ce que montre cet exemple, c'est qu'il importe d'appréhender l'œuvre de genre dans un réseau beaucoup plus large (intertextes, architextes, paratexte, images, etc.) qui en détermine le sens, faisant voler en éclats l'idée d'une unité propre au texte. L'œuvre sérielle - et donc l'œuvre de genre - est le produit d'une multitude d'énoncés.

22 Mais ce réseau de textes dépasse largement l'espace des productions littéraires pour toucher à l'ensemble des productions discursives et médiatiques. L'unité du récit vernien repose également sur la matérialité du support et sur les illustrations - et à ce niveau aussi (comme à celui du texte), on peut dire que son œuvre est une co-création avec Hetzel. De même, dans bien des récits populaires, l'illustration contribue largement à produire un effet de merveilleux scientifique quand les récits se contentent souvent d'intrigues aventureuses. Ce que cela signifie, c'est que les unités architextuelles circulent entre les médias et les modes d'expression, du texte au support, de l'écrit à l'image, mais 
aussi d'un mode d'expression à l'autre. On évoque par exemple dans les années 1930 théâtre et cinéma d'anticipation ${ }^{15}$, bien plus, on s'affranchit des limites médiatiques, comme lorsqu'on parle d'un «film d'anticipation à la manière de Wells " ${ }^{16}$, tout comme on parlait, pour une pièce de Jules Verne, d'un « roman scientifique $»^{17}$. Cela ne veut pas dire pour autant que les généricités cinématographiques ou théâtrales obéissent aux mêmes conventions que celles du roman : ainsi, quand le premier cinéma (Georges Méliès, Marcel Fabre) s'inspire du modèle vernien (et donc de ce qu'on pourrait identifier comme $\mathrm{du}$ " roman scientifique »), ce n'est pas pour sa dimension vulgarisatrice, mais pour ses qualités attractionnelles fort proches de celles de la prestidigitation ${ }^{18}$. De même, les adaptations théâtrales de Verne avaient beau se référer au "roman scientifique », elles délaissaient la question de la transmission vers les enfants pour exploiter les possibilités spectaculaires d'un théâtre à dispositifs et trucs. Mais si les différents médias et modes d'expression produisent leurs propres cohérences sérielles, ils manifestent une circulation transmédiatique des imaginaires génériques dont on doit toujours tenir compte lorsqu'on aborde ce type de questions.

De la même façon, il convient de considérer la circulation des imaginaires sériels au-delà des productions fictionnelles en tenant compte des échanges qui se produisent avec les discours non-fictionnels. Ainsi n'existe-t-il pas d'hétérogénéité entre les discours littéraires, savants et journalistiques. Et l'on ne peut comprendre les genres fictionnels sans les replacer dans les cohérences plus larges des discours médiatiques. Quand un journal comme Les Ailes, spécialisé dans l'aviation, ne cesse d'évoquer des "romans, anticipations à la Wells » ou des machines «à la manière de Jules Verne » lorsqu'il parle de prototypes d'avions ou de projets d'améliorations techniques ${ }^{19}$, il met en évidence une circulation des discours bien au-delà des seuls espaces des fictions narratives, et il témoigne d'une résonance des sérialités fictionnelles dans les imaginaires scientifiques et techniques et plus largement dans l'idéologie du progrès. Ainsi la trame des discours qui produisent l'unité du genre ne se limite pas aux textes qui commentent les œuvres, mais s'ouvre à ceux qui se réfèrent, explicitement ou implicitement, aux imaginaires du genre pour évoquer des sujets non littéraires. Fait-divers et roman policier, types de sujets scientifiques et récits d'anticipation (ou « romans scientifiques»), exploits d'un voyageur et roman d'aventures, témoignent que la littérature est un chaînon parmi d'autres du discours médiatique, et à travers lui, du discours social au sens où l'entend Marc Angenot 20. Cela revient à dire que la généricité offre une formulation du discours social, autrement dit que les genres structurent les imaginaires de la modernité, les mythes collectifs et les idéologies en donnant une forme lisible à leurs tensions et à leurs résolutions. Cette idée avait déjà été développée par Fredric Jameson ${ }^{21}$, mais ce qui apparait ici, c'est combien l'inconscient politique que les genres mettent au jour ne peut se saisir qu'à travers la restitution de la diversité des réseaux sériels entremêlés, des variations architextuelles dont ils sont l'expression, et des tensions sociales différentes dont ces variations rendent compte. C'est dans cette trame de discours variés que l'on peut saisir la signification sociale et culturelle des imaginaires génériques. En ce sens, l'étude des genres participe toujours d'une logique d'Histoire culturelle.

Ce lien entre généricité et discours social impose alors de penser la façon dont le genre restitue les sociabilités qui le portent et les rapports de force qu'il exprime. Nous avons déjà évoqué le second point, à travers l'opposition entre roman d'aventures extraordinaires (peu légitimé littérairement) et anticipation recherchant une légitimité middlebrow, ou à travers la valeur des textes produits par les modes de réception 
qu'induisent les supports et le champ littéraire. On peut prendre un exemple du premier point. Dans le premier chapitre d'Un drame au fond des mers, Richard Cortambert évoque une scène de sociabilité qui illustre les effets de cohérence générique induits par les logiques de sociabilité.

Ce jour-là, 15 juin 1866, la Société compait une partie de ses notabilités. On apercevait [...] M. Babinet, de l'Institut, qui fut, comme il ne l'ignorait pas, le plus spirituel, le plus érudit, le plus littéraire et le plus illustre des journalistes scientifiques. M. Philarète Chasles se trouvait placé à côté de Nadar [...] M. de La Landelle parlait, bien entendu, de l'aviation et de la mécanique, en romancier, avec M. Verne. M. Wilfrid de Fonvielle se lançait à corps perdu dans les nuages avec M. Flammarion. ${ }^{22}$

Le texte présente côte à côte diverses célébrités. Parmi celles-ci, un romancier clé, Jules Verne, et un certain nombre de vulgarisateurs ayant écrit des fictions, comme Wilfrid de Fonvielle ou G. La Landelle. Il met ainsi en scène un trait de la sociologie littéraire du roman scientifique, le fait qu'il est souvent écrit par des auteurs qui se présentent à la fois comme des romanciers et des savants. La nature du texte et ses usages ne peuvent se comprendre sans cette unité sociologique. Mais celle-ci à son tour ne peut s'interpréter indépendamment d'un questionnement sur le discours social. Les ambitions éducatives ont changé de cible, visant moins l'édification qu'un savoir positif; l'imaginaire scientifique est quant à lui largement lié à une logique de merveilleux profane présent dans bien d'autres domaines (qu'on a pu associer à un réenchantement du monde après le déclin du sacré) ${ }^{23}$. On citera, parmi ces domaines jouant sur l'émerveillement en exploitant la porosité avec la fiction, les Expositions Universelles, les démonstrations scientifiques, mais aussi par exemple la prestidigitation fictionnalisant les procédés scientifiques. Cette importance des spectacles de magie profane, bien repérée par During dans Modern Enchantments ${ }^{24}$, appartient à une série culturelle et médiatique hétérogène du roman scientifique; mais elle s'inscrit en revanche dans le même paradigme culturel du «merveilleux scientifique». C'est bien tout un système social, comme logique de sociabilité et modes de représentation du monde, qui porte le genre.

\section{Généricité et sérialité}

On voit dès lors que la question des genres suppose de traquer avant tout les effets de sérialisation des discours. Par sérialisation, nous entendons des logiques de séries qui font entrer en résonance des œuvres, produisant des attentes spécifiques. Ces séries ne se limitent pas aux séries génériques proprement dites. Il faut les croiser avec des séries discursives d'autre nature (les récits d'anticipation scientifiques avec les textes scientifiques; les récits de guerre future avec les textes de stratégie-fiction, les contes d'anticipation avec les esthétiques symbolistes, etc.). Il faut aussi les confronter aux autres séries médiatiques (en tenant compte des unités secondes: chaque média est producteur d'unité architextuelle, mais, au sein de chaque média, la variété des supports, des modes de distribution, des publics visés et des types de consommation produit des unités architextuelles secondes. Il faut encore croiser le genre avec des séries culturelles (comme celle des différents spectacles scientifiques, on l'a vu). Il faut enfin les croiser avec des séries sociodiscursives (l'assomption d'un Maurice Renard est par exemple indissociable d'une montée en puissance d'une littérature d'imagination middlebrow, sous l'influence revendiquée de la littérature anglo-saxonne, laquelle ne peut s'expliquer que par les transformations de la relation aux loisirs et à la lecture). 

Qu'est-ce qui reste de l'anticipation, dès lors qu'on en vient à distinguer une multitude d'unités architextuelles secondaires, souvent au croisement de séries médiatiques ou sociodiscursives, comme les « récits de guerre future », les « romans scientifiques publiés dans des volumes pour les étrennes", les "aventures étranges publiées dans des collections pour la jeunesse ", les " anticipations middlebrow », etc. ? Cette fragmentation n'est-elle pas contredite par l'affirmation que nous avons formulée en parallèle d'une circulation des architextes dans des médias, des formes discursives et des modes d'expression différents, au point de toucher à l'ensemble du discours médiatique et de structurer l'imaginaire social?

dépasser cette contradiction apparente, on peut faire appel à une opposition pensée par Louis Francoeur puis par André Gaudreault - au prix il faut le reconnaître d'une réorganisation de ces concepts ${ }^{25}$. Dans son étude sur le premier cinéma, Gaudreault oppose les "séries culturelles" au "paradigme culturel", le second englobant les premières. Les séries culturelles sont des structures de signification résultant d'un découpage cognitif du champ médiatique, les paradigmes culturels sont des structures englobantes rassemblant différentes séries dans une unité culturelle. Autrement dit, le paradigme culturel est un polysystème subsumant des séries culturelles différentes. Francoeur parle par exemple du polysystème culturel québécois et des séries le composant (littérature, peinture, art et tradition populaire du Québec); Gaudreault évoque de son côté le polysystème du spectacle de scène de la fin du XIXe siècle et ses unités sérielles de signification (café-concert, théâtre d'ombres, féérie, pantomime).

Il nous semble que cette opposition entre série et paradigme peut aisément être utilisée quand il s'agit de réfléchir aux mécanismes génériques. Si les différents médias partagent souvent les mêmes terminologies génériques et se réfèrent parfois aux mêmes prototypes, c'est bien qu'ils s'inscrivent dans un paradigme unifié, celui d'une culture médiatique comme régime dominant d'appréhension du monde et comme polysystème de représentation en interaction constante (puisque la culture médiatique est une culture transmédiatique). Ce polysystème produit du même coup des conventions qui circulent entre les médias, et donc aussi un ensemble d'unités architextuelles, génériques et transmédiatiques fonctionnant globalement. On voudrait pour preuve de ce processus l'effet qu'a eu le développement du cinéma sur la fixation des terminologies génériques si on en croit du moins la façon dont les terminologies génériques sont rapidement devenues moins souples à partir des années 1910-1920. De fait, des expressions comme récit policier, récit d'aventures, récit d'anticipation vont après la guerre se substituer à des formules plus descriptives ("grand roman d'amour et d'aventures", «Drame criminel», "aventures fantastiques », etc.). Il n'est pas impossible que cette fixation terminologique ait répondu à un besoin de compenser la diffraction des récits dans des médias et des modes d'expression différents en standardisant les moyens de les décrire. On peut même penser que la stabilisation des traits génériques répond aux besoins de posséder des outils permettant de rendre compte de cet imaginaire transmédiatique homogénéisé.

Mais on peut aller plus loin, et considérer que, dans la mesure où les imaginaires sériels se situent à l'intersection d'un axe synchronique (l'interdiscours) et d'un axe diachronique (les héritages sédimentés), alors ils participent d'une façon essentielle à une unité paradigmatique plus diffuse, celle d'un imaginaire social et d'un discours social auxquels leurs structures conventionnelles permettraient de donner des formes 
convaincantes. En ce sens, ils reflètent et structurent les positions des différents acteurs dans l'espace socioculturel. Faute de place, nous ne développerons pas cet aspect, mais il nous paraît évident qu'on peut expliquer par cette double fonction de reflet et de structure la tendance des genres médiatiques à adopter des positions dialogiques : pour les corpus qui nous intéressent, attraits et dangers de la science, futur hésitant entre fantasmes utopiques et visions dystopiques, oscillation entre rationalisme et lectures irrationnelles - merveilleuses - de la science, mise en valeur du génie occidental et des puissances primitives des sauvages, etc. Les genres mettent en scène des positions dialogiques parce que, productions collectives, ils reflètent les tensions entre les différents acteurs sociaux : mais ils résolvent aussi ces tensions, le plus souvent dans le sens d'un consensus. Dès lors, les mutations au fil du temps de ces tensions et de leur résolution nous donnent une image tout à la fois des rapports de force sociaux et des idéologies du temps. Le caractère dialogique des genres envisagés dans leur généralité la plus grande permet en retour de comprendre l'existence de séries génériques secondes, liées aux conditions matérielles de production et de réception des œuvres. De fait, on comprend à présent que ces unités secondes correspondent aussi à des fonctions différentes associées en contexte aux œuvres et aux genres, et donc des usages, des cohérences idéologiques et des effets de signification liés aux usages et aux logiques de communication.

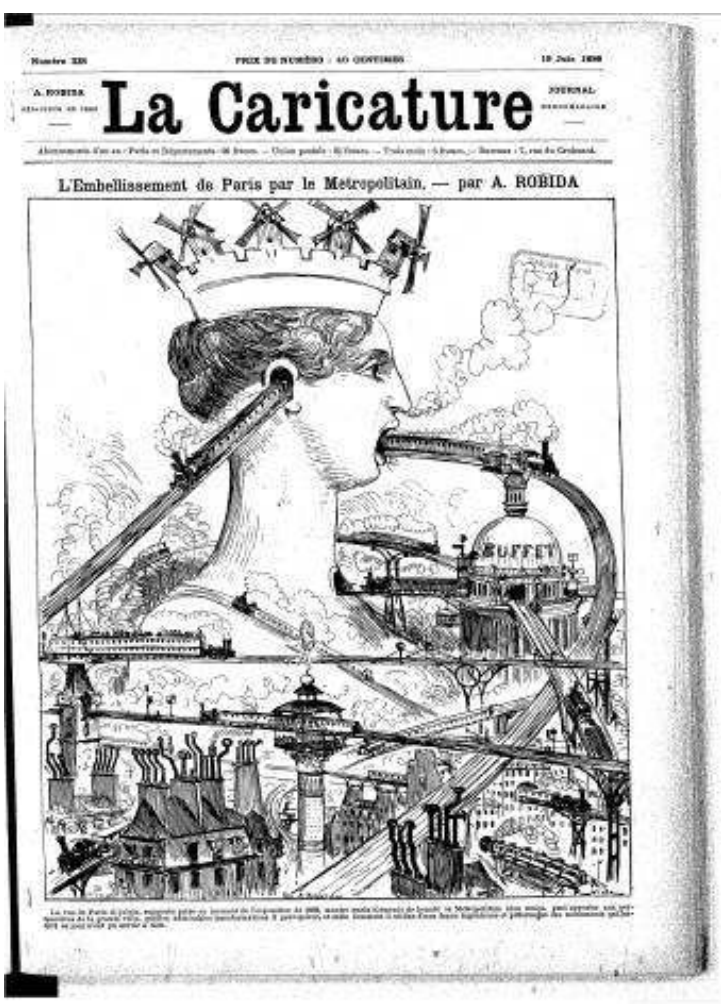

1. Robida, La Caricature, 19 juin 1886.

On pourrait encore ajouter d'autres dynamiques qui tendent à territorialiser les unités sérielles, par exemple les grands axes d'opposition qui structurent nos représentations mentales des productions culturelles (et qui déterminent donc les façons de penser les œuvres). On peut en rappeler quelques-unes, mais il y en a sans doute bien d'autres: fictif/ non fictif ; narratif/ discursif ; textuel/ visuel ; artistique/ commercial ; etc. Dans ce cas encore, en produisant des effets de cohérence, de telles distributions des œuvres tendent à produire des effets de spécialisation des conventions génériques. Certes, les 
articles journalistiques d'anticipation (ceux par exemple qui évoquent dans les années 1880-90 la vie au XXe S.) ou les descriptions de machines de la fin du XIXe siècle interagissent avec les œuvres fictionnelles qui investissent les mêmes sujets. De même, les images et les textes représentant des machines extraordinaires retrouvent des stéréotypes communs, tout comme les œuvres à prétentions artistiques, les productions distractives de la petite presse ou les fictions narratives et les fictions mimétiques, etc.; mais il est clair que chacune de ces sous-séries interagit en parallèle autant avec les productions qui occupent le même territoire qu'elles, même quand elles parlent de tout autre chose. Quand Albert Robida choisir de stigmatiser la société moderne à travers des extrapolations caricaturales (image 1), il utilise des motifs que l'on rencontre dans toute la littérature d'anticipation de l'époque, mais ses procédés sont aussi ceux de toute la petite presse satirique: usage du décalage entre texte et image, exagération, ironie, parodie, recours à des cibles repérées à l'époque (la libération de la femme, la modernité artistique, les valeurs de la bourgeoisie, etc.). La façon qu'il a de représenter ces sujets ressemble à celle qu'il emploie quand il caricature la politique (image 2) ou le cirque à l'américaine. Autrement dit, la logique de Robida est celle de la caricature. Simplement, prenant pour sujet la question de la modernité et de ses travers, il l'exagère (comme il exagère les vogues artistiques, par exemple au Salon de 1887, etc.). Or, exagérer caricaturalement la modernité, ce mouvement d'arrachement du passé au profit de l'avenir, c'est produire des images exagérément modernes - c'est-à-dire des images d'anticipation. Dès lors, étudier Robida avant tout sous l'éclairage de l'anticipation, c'est perdre de vue la signification contextuelle de son œuvre, profondément liée aux logiques de la presse satirique.

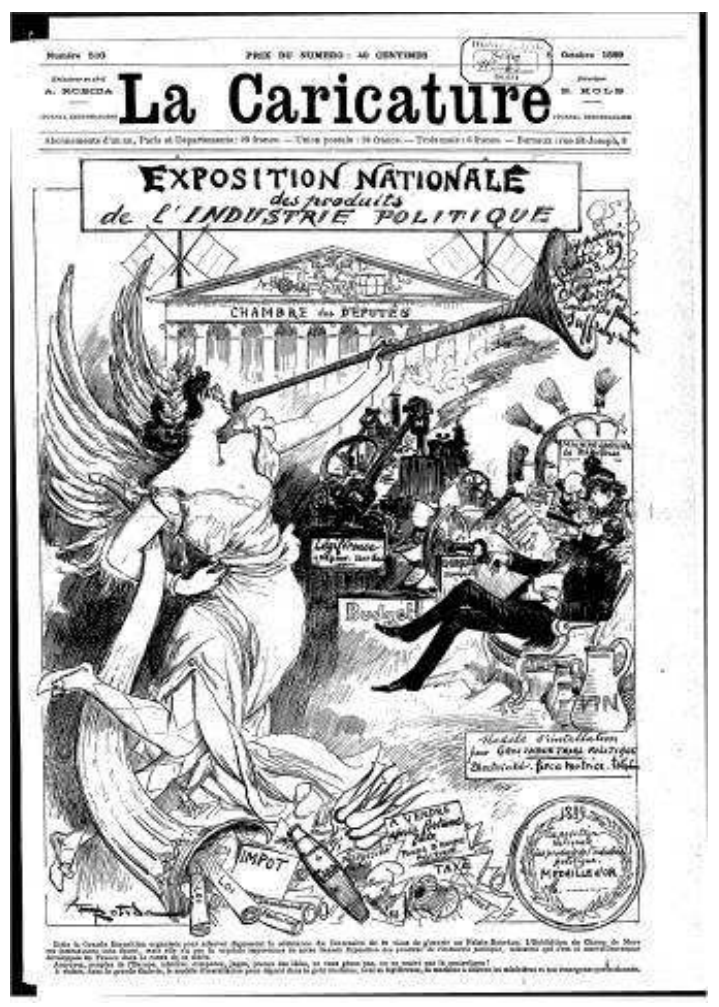

2. Robida, La Caricature, 5 octobre 1889.

On peut tirer une dernière conclusion de ces remarques : la fragmentation des genres en multitude de sous-séries peut donner l'impression d'un délitement du genre dans autant 
de distinctions byzantines. Mais elle démontre surtout que les séries architextuelles gagnent à être pensées à partir de leurs situations socioculturelles d'énonciation. Cela signifie que le genre se construit toujours à partir d'une œuvre ou d'un discours, et qu'il n'en existe pas d'unité qui transcenderait ses actualisations. Dès lors, chaque auteur, chaque œuvre propose une définition qui ne recoupe que partiellement les autres. De même en est-il pour chaque lecteur, dont les attentes sont déterminées par sa relation au genre, pour chaque commentateur, critique, journaliste, enfin pour chaque éditeur ou publicitaire. Tous offrent des interprétations du genre et, dans un même mouvement, renforcent les séries qui coïncident avec leur interprétation. Ainsi, ils dialoguent avec l'interdiscours et ses héritages, qu'ils reformulent, et auquel ils participent suivant leurs intérêts propres et leur influence dans l'espace public. Cette multitude de positions tend à objectiver les séries culturelles et les architextes, mais selon une modalité toujours flottante, avec les contradictions que cela implique. On voit qu'une telle perspective s'inscrit dans cette logique de «ressemblance de famille» définie par Ludwig Wittgenstein ${ }^{26}$. Comme l'avait montré ce philosophe, un concept peut renvoyer à des objets extrêmement hétéroclites, au point de ne pouvoir se voir associé à une définition unifiée; et en même temps, tous les objets qu'il rassemble possèdent une "ressemblance de famille ». Ainsi, Wittgenstein souligne qu'il n'y a pas nécessairement de traits unitaires généraux liant les différents objets rassemblés dans une catégorie transcendante, mais plutôt un ensemble de liens conceptuels dont la pertinence n'est toujours que partielle et variable. La conséquence en est que le regard que nous portons sur le genre changera suivant le lieu d'où nous l'observons : chaque œuvre privilégie certaines séries génériques et architextuelles, définit des héritages et des cohérences; chaque série met l'accent sur des traits architextuels au détriment d'autres, et valorise des figures prototypiques plutôt que d'autres. Il n'y a réellement d'unité du genre que relative, déterminée à partir du point d'où on l'observe. Chercher à lui opposer une définition transcendante c'est perdre la mécanique productive du genre.

On peut dès lors repenser les perspectives transcendantes et anachroniques qui prévalent le plus souvent dans l'étude des genres. Celles-ci se révèlent comme les expressions d'une position située et d'un dialogue avec les séries culturelles (dans une perspective synchronique) et les héritages discursifs (dans une perspective diachronique) au même titre que les autres positions. Si les perspectives transcendantes ou anachroniques ont longtemps prévalu, c'est simplement qu'elles s'appuyaient sur un consensus académique imposant une lecture située comme la bonne lecture, dans une logique qu'on pourrait qualifier de bourdieusienne (au sens où elle repose sur un assentiment collectif à un modèle distinctif relativement homogène). Si une telle position est devenue problématique, c'est que le modèle bourdieusien s'est en partie effondré et que d'autres systèmes de hiérarchisation, qui ont toujours existé mais ont été minorés par les autres positions dominantes, entrent désormais en concurrence avec les discours académiques et les discours critiques légitimés, au point de redéfinir par exemple la nature des modèles évaluatiff ${ }^{27}$. Ces autres systèmes de hiérarchisation sont par exemple ceux de l'industrie culturelle, des médias de masse, de communautés de lecteurs obéissant à des principes analytiques propres - communautés sous-culturelles et contre-culturelles, groupes homogènes d'amateurs, publics liés par des caractéristiques d'âge, de sexe, de région, de classe sociale, etc.

Dès lors, ressaisir les perspectives transcendantes ou anachroniques comme des positions situées qui ne disent pas leur nom (autrement dit, comme de l'idéologie) revient à 
déconstruire notre propre impensé disciplinaire fondé sur un consensus sur les corpus, les modes de lecture des œuvres et les définitions à partir desquelles les textes sont appréhendés. Or, cet impensé est ce qui explique aussi le déclin de notre discipline. Nous sommes devenus marginaux parce que notre définition de la culture est elle-même devenue marginale. De fait, l'idée d'une culture comme grande culture (qui faisait encore consensus à la fin des années 1970 et sur laquelle reposent encore largement les études littéraires) a cédé la place à une définition de la culture comme ensemble de pratiques d'une collectivité. Lors même qu'elle prétend prendre la culture pour objet central, notre discipline se propose d'évoquer celle-ci suivant une définition largement désuète. Dépasser ces perspectives disciplinaires s'impose comme une mue nécessaire, et ce, aussi bien dans l'étude de la généricité que dans les autres champs d'analyse, puisqu'il s'agit d'une solution pour engager à nouveau le dialogue avec ce que nous avons perdu de vue, et qui est pourtant notre seul objet : la culture.

\section{NOTES}

1. Cette réflexion, comme les autres analyses portant sur les anticipations de la période 1860-1940, prend appui sur les travaux accomplis dans le cadre de l'ANR anticipation.

2. Par exemple dans Comoedia, 1910, à propos du roman de Gaston Dumestre, Monsieur van Grippenbergh.

3. Florence Goyet, La Nouvelle, 1870-1925 ; description d'un genre à son apogée, Paris, PUF, 1993.

4. Paul Veyne, Comment on écrit l'Histoire, Paris, Seuil, 1971.

5. Sur cette notion de référent prototypique, voir infra.

6. Congrès scientifique de France, septembre 1845.

7. Jean-Marie Schaeffer, Qu'est-ce qu'un genre littéraire ? Paris, Seuil, « Poétique », 1989.

8. Denis Mellier, «Steampunk, transfictionnalité et imaginaire générique (littérature, bandes dessinées, cinéma) », in René Audet et Richard Saint Gelais, La Fiction, suites et variations, Québec et Rennes, Nota Bene et PUR, 2007.

9. Michael Saler, As If, Modern Enchantment and the Literary Prehistory of Virtual Reality, Oxford, Oxford University Press, 2012.

10. Matthieu Letourneux, "Quand arrivera la guerre de demain - bientôt, je veux l'espérer ; Récits de guerre future, généricité et discours social ", Nineteenth-Century French Studies, 43, printemps-été 2015. Pour ce qui concerne le poids dans le discours social des imaginaires patriotiques et revanchards à la Belle Époque, on lira Marc Angenot, 1889, un état du discours social, Longueuil, Le Préambule, «L'Univers du discours », 1989.

11. Marie-Eve Thérenty, « Pour une poétique du support », Romantisme, 2009, $\mathrm{n}^{\circ} 143$

12. Howard Becker, Les Mondes de l'art, Paris, Flammarion, 1988.

13. Par exemple La Vaulx et Galopin, Le Tour du monde en aéroplane, 1905, Galopin, Le Tour du monde en sous-marin, 1924, Christophe, Le Tour du monde en 80 heures, par l'as Perge Firmin, 1920, Norbert Sevestre, Le Tour du monde en 14 jours, 1926, H. de Graffigny, Le Tour du monde en auto, 1909-1910, etc.

14. M. Letourneux, « Répétition, variation...et autoplagiat ; Les pratiques d'écriture de Jean de La Hire et la question des stéréotypes dans les genres populaires ", Littérature et stéréotypes, Loxias, 17 (http://revel.unice.fr/loxias/index.html?id=1699) 
15. C'est le cas par exemple de Sensorium Limited de Maurice Tumerelle (1931), présenté comme une "pièce d'anticipation » ou du film Point ne tueras de Maurice Elvey, «film d'anticipation » (1929).

16. L'Écho d'Alger, 1931.

17. Critique du Voyage à travers l'impossible, Le Figaro, 26 novembre 1886.

18. Sur les logiques attractionnelles du premier cinéma, voir André Gaudreault, Cinéma et attraction, Pour une nouvelle histoire du cinématographe, Paris, CNRS Éditions, 2008.

19. «Romans, dira-t-on, anticipations à la Wells » («L'Organisation de l'État et le danger aérien », 25 septembre 1930) ; «Certains souriront et parleront d'anticipations à la Wells» («La Défense active, une coûteuse illusion?» 12 mai 1932) - dans Les Ailes, les références se comptent par dizaines, au point d'apparaître comme un tic de langage du périodique.

20. Marc Angenot, 1889, op. cit..

21. Fredric Jameson, L'inconscient politique, Paris, Questions théoriques, "Saggio Casino », 2012 [1981].

22. Richard Cortambert ; Un Drame au fond de la mer, Paris, Decaux, 1876.

23. Michael Saler, As If, op. cit., et Joshua Landy et Michael Saler (dir.), The Re-Enchantment of the World: Secular Magic in a Rational Age, Stanford, Stanford University Press, 2009.

24. Simon During, Modern Enchantments, Cambridge, Harvard University Press, 2002.

25. Louis Francoeur, "Quand écrire c'était agir: la série culturelle québécoise au XIXe siècle », Voix et images, 6, 3, 1981 ; et André Gaudreault, Cinéma et attraction, op. cit.

26. Ludwig Wittgenstein, Investigations philosophiques, in Tractatus logico-philosophicus, suivi d'investigations philosophiques, Paris, Gallimard, « Tel », 1961.

27. Sur ces questions, voir Hervé Glevarec, La Culture à l'ère de la diversité, Paris, éditions de l'aube, 2013.

\section{INDEX}

Mots-clés: genre, histoire littéraire, sérialité, culture médiatique, culture de masse, culture populaire

\section{AUTHOR}

\section{MATTHIEU LETOURNEUX}

Matthieu Letourneux est Professeur à l'Université Paris Ouest Nanterre-La Défense et membre du CSLF-PHISTEM. Spécialiste des cultures sérielles et médiatiques des XIXe et XXe siècles et de la littérature pour la jeunesse, il a publié Le Roman d'aventures, 1870-1930 (PULIM, 2010), La Librairie Tallandier, Histoire d'une grande maison d'édition populaire (1870-2000) (avec J.-Y. Mollier, Nouveau Monde, 2011), Fantômas ! Biographie d'un criminel imaginaire (avec L. Artiaga, Les Prairies ordinaires, 2013) et Cinéma, premiers crimes (avec A. Carou, Paris Bibliothèques, 2015). Il a dirigé ou codirigé des publications collectives sur les littératures de jeunesse ou les cultures médiatiques. Il a réédité une dizaine d'ouvrages, parmi lesquels, chez Phébus, Le Coureur des bois de Gabriel Ferry, au Masque, On vole des enfants à Paris, de Louis Forest, et chez Bouquins, des 
œuvres de Gustave Aimard, Emilio Salgari, Eugène Sue et la série Fantômas (avec L. Artiaga). Il est rédacteur en chef de Belphégor (http://belphegor.revues.org/). 